\title{
Special Section on New Challenge for Internet Technology and its Architecture
}

The character of the Internet has drastically changed from experimental networks for a small number of technical experts into a common infrastructure for people in the world. The importance of Internet technology will become further evident in a ubiquitous network era, when everything is equipped with network feature and high-speed network service is freely provided anytime, anywhere.

Conventional approaches for academic research on the Internet are strongly tied to Western reductionism and tend to narrow the scope of research into specific fields, and they sometimes fail to grasp the Internet as architecture. There are so many activities and technical developments that have occurred outside academic societies. It has passed six years since the Internet Architecture (IA) Technical Committee was established to improve such situation.

Based on these activities, this special section on "New Challenge for Internet Technology and its Architecture" is planned as the fifth one of the series on Internet Technology. For the call for the papers, 53 papers were submitted to the section, and 26 of them are from abroad. After careful review process the editorial committee accepts 17 papers for publication. The accepted papers include recent hot topics in various technical developments, and we believe that all of them are worthy of being called new challenges.

Finally the editorial committee would like to express their sincere appreciation to all authors for their valuable contributions and to all reviewers for their cooperation in completing the review process under the tight schedule.

Member of editorial committee of this special section are:

Guest Editor-in-Chief: Yasuo Okabe (Kyoto University)

Guest Editor: Hideki Sunahara (NAIST)

Guest Associate Editors:

Katsuyoshi Iida (Tokyo Inst. of Tech.), Norihiro Ishikawa (NTT DoCoMo), Takashi Imaizumi (Chiba Univ.), Keisuke Uehara (Keio Univ.), Koji Okamura (Kyushu Univ.), Akio Kawabata (NTT), Masatoshi Kakiuchi (NAIST), Toshihiko Shimokawa (Kyushu Sangyo Univ.), Kenichi Nagami (Intec NetCore), Motonori Nakamura (NII), Yutaka Nakamura (Kyushu Inst. of Tech.), Yoshifumi Nishida (Sony CSL), Koji Nishimura (Hiroshima Univ.), Kaori Maeda (Hiroshima City Univ.), Katsuyuki Yamazaki (Nagaoka Univ. of Tech.), Kenichi Yoshida (Tsukuba Univ.)

Yasuo Okabe, Guest Editor-in-Chief

Yasuo Okabe (Member) received the B. Engineering and M. Engineering from the Department of Information Science, Kyoto University in 1986 and 1988, respectively. $\mathrm{He}$ joined Faculty of Engineering, Kyoto University in 1988 as an Instructor, and received Ph.D. in Engineering in 1994. From 1994 he was an Associate Professor of Data Processing Center, Kyoto University, and moved to the Graduate School of Informatics, Kyoto University in 1998. Since 2002 he has served Academic Center for Computing and Media Studies, Kyoto University, as a Professor, and has been a Professor in charge of Division of Intelligence Science and Technology, Graduate School of Informatics. As a visiting professor of National Institute of Informatics (NII), he has been conducting UPKI (Inter-University PKI) project for Cyber Science Infrastructure since 2005. He was the project leader of "Self-organizing Network Infrastructure" research project in "Intelligent and Dynamic Inter-networking" research field of JSPS Research for the Future Program. His current research interest includes multi-

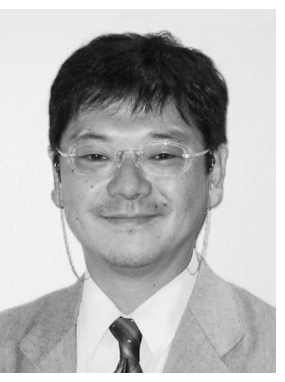
media Internetworking, network security and distributed systems. He is a vice chair of 163 rd Committee on Internet Technology (ITRC), JSPS University-Industry Cooperative Research Committees. He is the former chair of Internet Architecture (IA) Technical Committee of IEICE. He is a member of IPSJ, ISCIE, JSSST, IEEE, ACM, and EATCS. 